\title{
Towards Application of Bioactive Natural Products Containing Isoprenoids for the Regulation of HMG-CoA Reductase-A Review
}

\section{Sepideh Pakpour}

Department of Biology, University of British Columbia, Kelowna, Canada.

Email: sepideh.pakpour@ubc.ca

Received March $1^{\text {st }}, 2013$; revised April $2^{\text {nd }}, 2013$; accepted May $1^{\text {st }}, 2013$

Copyright (C) 2013 Sepideh Pakpour. This is an open access article distributed under the Creative Commons Attribution License, which permits unrestricted use, distribution, and reproduction in any medium, provided the original work is properly cited.

\begin{abstract}
Recognition of the biological properties of numerous "natural products" has fueled the current focus of this field, namely, the search for new drugs, antibiotics, insecticides, and herbicides. Based on their biosynthetic origins, natural products can be divided into three major groups: the isoprenoids, alkaloids, and phenolic compounds. Isoprenoids are structurally the most diverse group of secondary natural metabolites with different roles in the growth, development, and reproduction of a diverse range of prokaryotic and eukaryotes cells. Mevalonate and 2-C-methyl-D-erythritol 4phosphate (MEP) pathways are known to be responsible for biosynthesis of numerous isoprenoids. HMG-CoA reductase is a rate-determining enzyme in mevalonate pathway, producing intermediates such as farnesyl and geranylgeranyl pyrophosphates, which lead to by-products such as cholesterol. Earlier studies have demonstrated that the inhibition of HMG-CoA reductase is one of the most effective approaches for treating hypercholesterolemia and eventually cardiovascular disease (CVD). Statins are HMG-CoA reductase inhibitors and the most prescribed group of drugs worldwide in treating hypercholesterolemia; however the application of this group of drugs may be expensive and has side effects including rashes and gastrointestinal symptoms. For these reasons, there is an important need to examine the viability of natural products as an alternative to statin treatment. This article is a review of different aforementioned areas with a focus on isoprenoids that can be used for the regulation of HMG-CoA reductase.
\end{abstract}

Keywords: Isoprenoids; HMG-CoA Reductase; Mevalonate Pathway; Hypercholesterolemia

\section{Introduction}

Primary metabolites are compounds that have important role in nutrition and essential metabolic pathway inside the plants, while natural (secondary) products do not participate directly in growth and development but have ecological roles between the plants and the environment including: 1) protection against herbivory and microbial infections; 2) attractants for pollinators and seed dispersing animals; and 3) act as allelopathic agent to suppress the growth of plant by a toxic released from a nearby plant of the same or another species [1-3]. Plant natural products are divided into three major groups: 1 ) isoprenoids, 2) alkaloids, and 3) and phenolic compounds, based on their biosynthetic origins.

This article reviews the isoprenoids groups with an interest on discussing their potential effects for treating hypercholesterolemia and cardiovascular disease (CVD). Namely, in Section 2, the isoprenoids' general definition, classification and synthesis with a focus on mevalonate pathway, as it is the main pathway for the production of cholesterol, are discussed. In Section 3, after an introduction of cholesterol, its different forms and biosynthetic pathway as well as regulation are presented. For the latter, in Section 4, the focus is on the review of HMG-CoA reductase and its regulation since it is the rate-determining enzyme in the mevalonate pathway. Finally, in Section 5, a summary along with potential future developments is presented for exploring other natural products, including those of essential oils of plants as alternatives towards statin treatments.

\section{Isoprenoids}

Isoprenoids are a large and highly diverse group of natural products. Nearly 35,000 isoprenoids, with largely varying structures and functions, have been identified in viruses, bacteria, fungi, yeast, plants, and mammals $[4,5]$. 
In plants, some isoprenoids are vital and moderately universal primary metabolites that act as growth promoters (e.g. abscisic acid, giberllines, cytokinins) or are essential in photosynthesis (e.g. caratenoid). Also, a group of plants' isoprenoids are secondary metabolites, such as antimicrobial compounds (phytoalexines) or plant essential oils and natural rubber [6,7]. Moreover, it has been demonstrated that some biologically active compounds, that are derived from intermediates of the isoprenoid pathway (e.g., sterols, oxysterols, farnesol, geranylgeraniol, diphosphate derivatives of isopentenyl (IPP), geranyl (GPP), farnesyl (FPP), geranylgeranyl (GGPP), and presqualene), have a role on the transcriptional and post transcriptional regulations of specific genes $[5,8]$.

Isoprenoids are classified into different groups according to the number of carbon they contain as follows: 1) hemiterpenes (C5H8); 2) monoterpenes ( $\mathrm{C} 10 \mathrm{H} 16) ; 3)$ sesquiterpenes $(\mathrm{C} 15 \mathrm{H} 24)$; 4) diterpenes $(\mathrm{C} 20 \mathrm{H} 32) ; 5)$ triterpenes $(\mathrm{C} 30 \mathrm{H} 48) ; 6)$ tetraterpenes $(\mathrm{C} 40 \mathrm{H} 64)$; and finally 7$)$ Polyterpenes $(\mathrm{C} 5 \mathrm{H} 8) \mathrm{n}$. All isoprenoids are originated from the universal C5 precursor isopentenyldiphosphate (IPP) and its allylic isomer dimethylallyldiphosphate (DMAPP), and are synthesized through two different biosynthetic pathways: 1) the classical acetate/mevalonate pathway in the cytosol and ER which is found in eukaryotes, archaea, and some bacteria (Staphylococcus aureus); 2) the nonmevalonate pathway or 2-C-methyl-D-erythritol-4-phophate/1-deoxy-D-xylulose 5-phosphate pathway (MEP/ DXP pathway) operating in the plastids which is the major pathway used in bacteria and plants [6,9-11]. The existence of the mevalonate pathway has been known for many years, while it has been about only 15 years since the MEP pathway has been discovered as another route of the biosynthesis of IPP and DMAPP [12]. Isoprenoids synthesis can be divided into four stages (Figure 1): 1) construction of the universal C5 precursor isopentenyl pyrophosphate (IPP) and its isomer dimethylallyl pyrophosphate (DMAPP); 2) condensation of IPP and DMAPP in a "head-to-tail fashion" by prenyltransferases to form the higher order isoprenoid building blocks, geranyl pyrophosphate (GPP; C10), farnesy 1 pyrophosphate (FPP; C15), and geranylgeranyl pyrophosphate (GGPP; C20); 3) conversion of GPP, FPP and GGPP by terpene synthases to the parent structure of the respective terpene groups; and finally, 4) transformation cyclization, and/or carboxylation of the parent structure to various derivatives of the basic isoprene groups. This step in most cases occurs in the cytosol [2].

\subsection{Mevalonate Pathway}

Isoprenoids that are crucial for diverse cellular function, ranging from cholesterol synthesis to growth control, are produced by mevalonate pathway $[8,9]$. Synthesis of each IPP in mevalonate pathway requires three molecules of acetyl-CoA, and this pathway starts with two steps condensation of these molecules of acetyl-CoA using two different enzymes (Thiloase and Hydroxymethyl-glutarilCoA synthase) to form a 3-Hydroxy-3methyl-glutarylCoA. Subsequently, the HMG-CoA molecule is reduced by HMG-CoA reductase to form a Mevalonic acid. This process continues by two subsequent phosphorilations of mevalonic acid using Mevalonate kinase and Phosphomevalonate kinase. Finally, phosphorylation/decarboxylation occurs using mevalonate diphosphate decarboxylase to yield an IPP, followed by an IPP isomerase that keeps the balance between IPP and its allelic isomer, DMAPP. In plants, Mevalonate pathway has more branch pathways than in most other organisms in order to produce a various number of isopronoid products [7].

All enzymes involved in this pathway are the site of drug's action [15], and the evidence suggests that HMG -CoA reductase is the rate-determining enzyme in mevalonate pathway witch catalyzes the conversion of hydroxymethylglutaryl-coenzymeA into mevalonate [16].

\section{Cholesterol}

Cholesterol (C27) is the major sterol in mammals and a byproduct of mevalonate pathway [17] where 19 enzymes are involved. All these enzymes including HMGCoA reductase exist in the steroidogenic cells [18]. Cholesterol biosynthesis is divided into two sections: 1) squalene portion where cholesterol biosynthesis starts by the head-to-head condensation of two molecules of FPP to produce presqualene diphosphate, then followed by an NADPH reduction step to produce squalene (C30); and 2) post-squalene portion where the squalene is epoxidized to form oxidosqualene, followed by a cyclization to produce lanosterol. Finally, lanosterol is reformed to produce cholesterol by loosing three methyl groups (C4, C14), and reducing the side-chain double bond (Figure 2) [4, $18,19]$.

Cholesterol has numerous crucial functions in human body. It is a key compound in cell membranes which can control the membrane over a range of physiological temperatures. Vitamin D is one of the cholesterol derivatives, and also, the human body uses the cholesterol as a precursor for the synthesis of bile acids, which are important for the digestion of dietary fats and fat-soluble vitamins. Finally, another important function of cholesterol is to serve as a substrate for the synthesis of all steroidal hormones including sex hormones, glucocorticoids and mineralocorticoids $[13,20]$. As a result, an obstruction in cholesterol biosynthesis may lead to multiple congenital anomalies and mental retardation [18]. 
Two forms of cholesterol are found in cells and plasma: free cholesterol which mainly spread in the plasma membrane, and to a lesser level in intracellular organelle; the second principal form of cholesterol is cholesterol esters, where cholesterol is esterified to long chain polyunsaturated fatty acids and to a lesser level of sulfate.

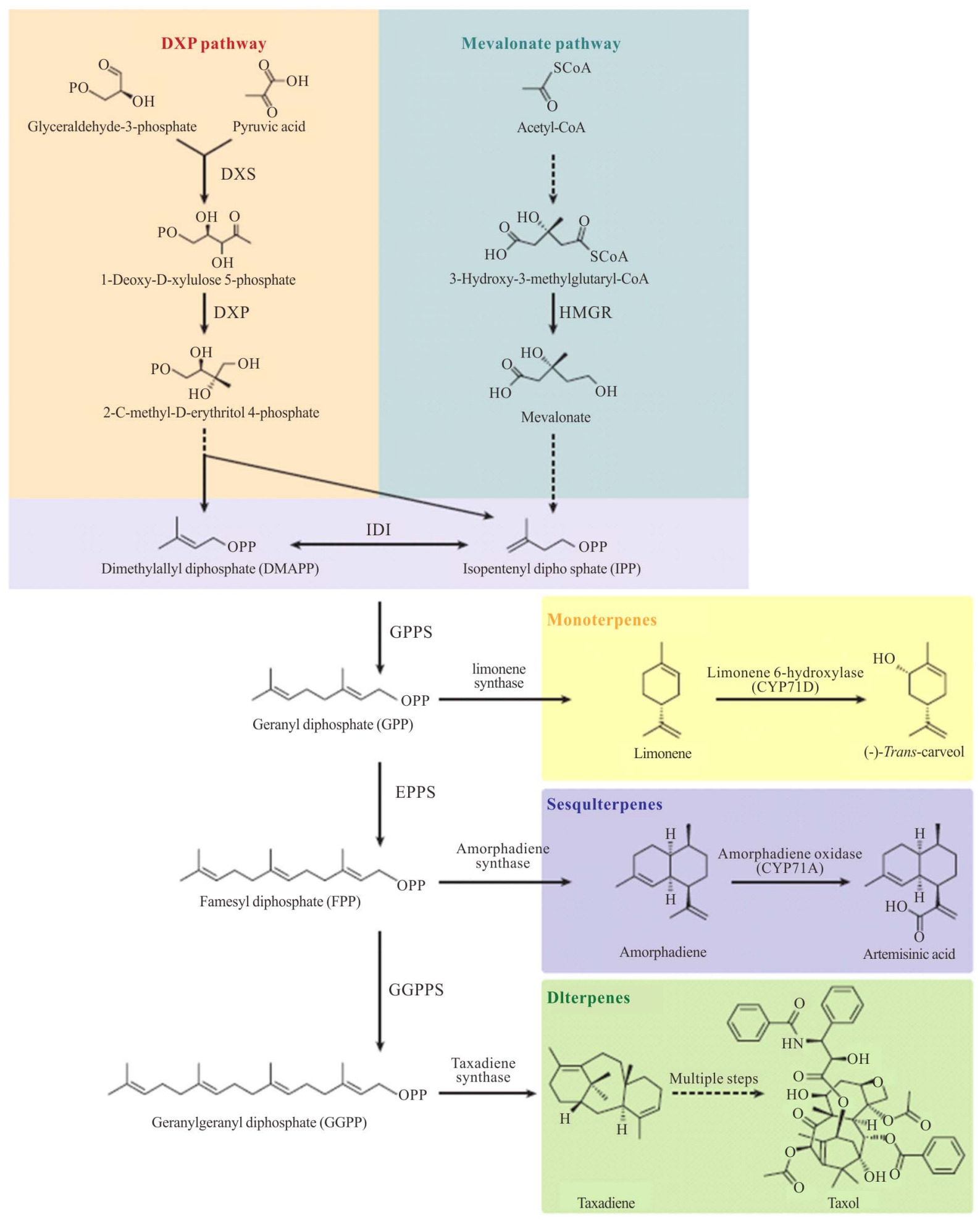

Figure 1. Schematic representation of isoprenoid biosynthetic pathways and products in different stages [14]. 
A
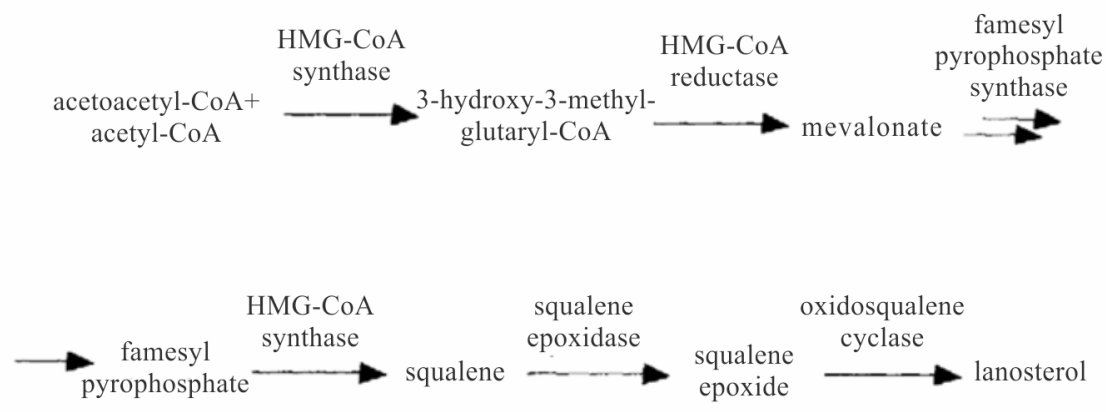

B

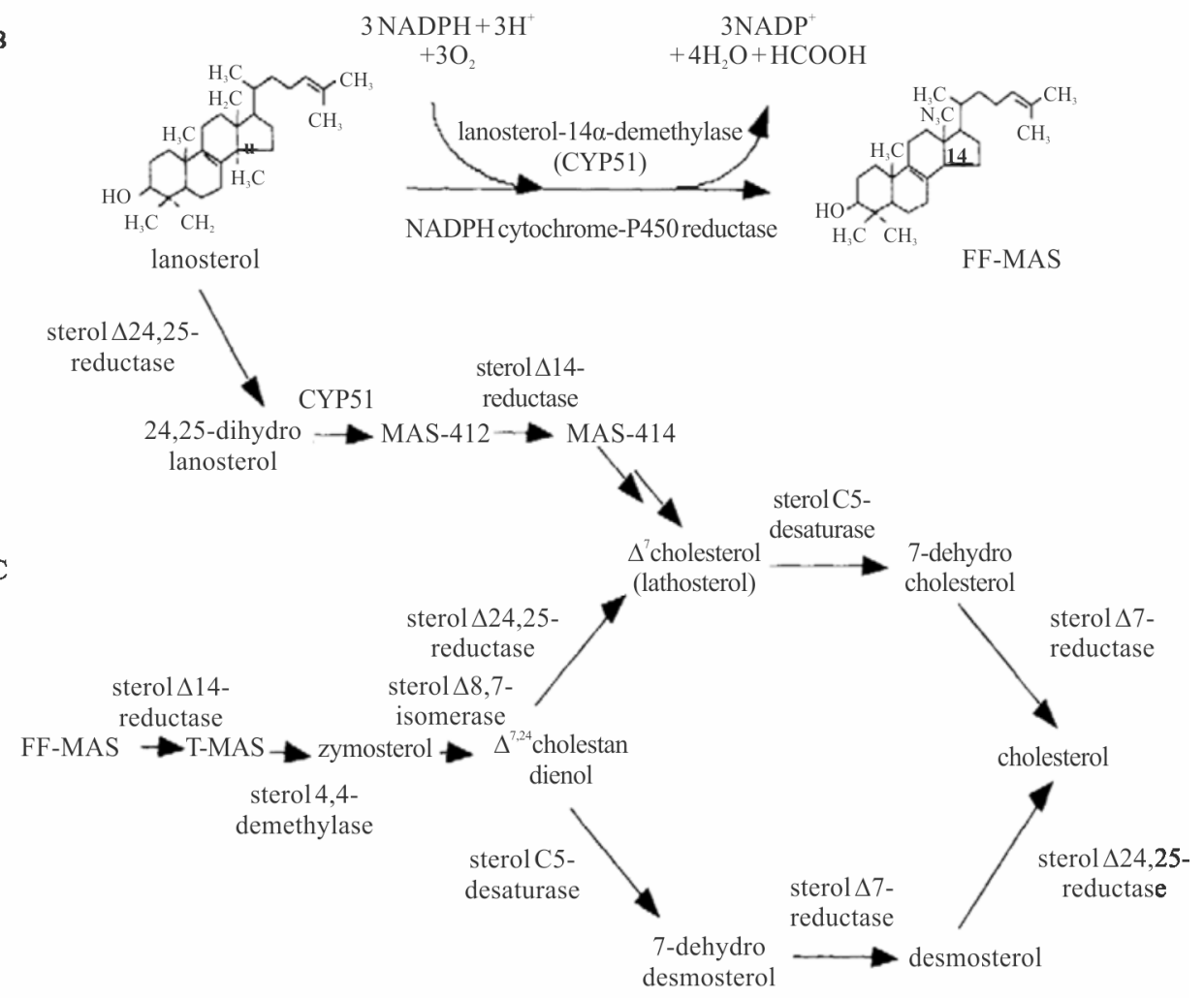

Figure 2. Overview of the cholesterol biosynthetic pathway. (A) Synthesis of lanosterol, (B) Synthesis of FF-MAS from lanosterol, (C) Conversion of MAS sterols to cholesterol [18].

This form of cholesterol is the main depots of surplus cellular sterol [18]. Cholesterol is insoluble in water and circulated by four different lipoproteins including chylomicron (CM), very low-density lipoprotein (VLDL), low-density lipoprotein (LDL), and high-density lipoprotein (HDL) in the blood [21]. Cholesterol ester is $60 \%$ $80 \%$ of total cholesterol content of the aforementioned lipoproteins [18]. The dietary cholesterol is absorbed through following steps and is then carried by chylomicron (CM) to adipose tissue and skeletal muscles: 1) first, cholesterol are transported from lumen to the intestine absorptive cells (enterocytes) via NPC1L1; 2) then, cholesterol is converted to cholesterol ester (CE) by using intestinal acyl coenzyme A: cholesterol acyltransferase 2
(ACAT2); 3) the latter product is packed into chylomicrons (CM) by using microsomal triacylglycerols (MTP); and 4) finally it is transferred into blood through the lymphatic system. The unabsorbed cholesterol is returned to the lumen of the intestine by employing ATP binding cassette transporters (ABCG5 ABCG8) [21-23]. LDL is produced in plasma and is the major cholesterol carrier in the blood which provides cholesterol to those tissues that need it. In contrast, HDL eliminates unwanted cholesterol from peripheral tissues back to the liver and have an important role in keeping cholesterol homeostasis in the plasma [13].

Heart disease is the second main cause of death in the world; 6 million people have died of cardiovascular dis- 
ease (CVD) in 2008 according to the World Health Organization (WHO) estimation, which is $10.8 \%$ of all death worldwide in the same year

(http://www.who.int/mediacentre/factsheets/fs310/en/ind ex.html). Also, heart disease is one of the main leading causes of death for Canadians; being responsible for $22 \%$ of the 235,217 deaths in Canada in 2007

(http://www.statcan.gc.ca/dailyquotidien/101130/dq1011 30b-eng.html).

Aging, body weight, blood pressure, smoking and drinking are the main factors which can raise the risk of CVD [24]. Also, studies demonstrated that there is an association between plasma cholesterol concentration and coronary heart disease where the over-accumulation of cholesterol within the body increases the risk of the cardiovascular disease [20].

\subsection{Regulation of Cholesterol Biosynthesis}

Cells gain cholesterol via two different mechanisms: 1) synthesis of endogenous cholesterol from acetyl coenzyme A (CoA) via mevalonate pathway; and 2) the cellular uptake of circulating plasma low-density lipoprotein (LDL) which enters the cell by receptor-mediated endocytosis (exogenously) $[25,26]$. These internal and external sources are balanced through feedback regulation of HMG-CoA synthase, HMG-CoA reductase as well as LDL receptors, in order to avoid sterol over-accumulation. In the presence of LDL, activities of aforementioned enzymes are $90 \%$ lower and mevalonate pathway is restricted to produce non-sterol end products. In contrast, in the absence of LDL, HMG-CoA synthase and HMG$\mathrm{CoA}$ reductase are highly active to produce cholesterol as well as non-sterol products. In the presence of sterol, the mevalonate pathway shunts into the biosynthesis of nonsterol compounds because the enzymes of non-sterol pathways have higher affinities than those of the sterol pathway $[5,8]$.

It has been illustrated that the higher fruit and vegetable intake results in the lower total cholesterol and lowdensity lipoprotein cholesterol (LDL-C); however it does not have any effect on the serum total cholesterol (TC) or HDL-C concentration $[27,28]$. In contrast, other studies illustrated that the consumption of functional foods containing high level of phytosterols resulted in the inhibition of intestinal NPC1L1, subsequently reducing the cholesterol absorption, and finally decreasing serum total cholesterol (TC) and low density lipoprotein (LDL) concentration $[29,30]$.

Generally, cholesterol-lowering agents can be categorized into five major types: acyltransferase (ACAT) inhibitors, LDL receptor activators, acyl-CoA: cholesterol cholesterol-bile acid absorption inhibitors, CETP (a plasma glycoprotein, which facilitates the transmission of $\mathrm{CE}$ from HDL to LDL) inhibitors, and HMG-CoA reductase inhibitors [13].

It has been shown that the tripenic acid [3], soya bean [31], naringenin as a citrus bioflavonoid [32], and conjugated linoleic acid [31] are effective in reducing ACAT activity and decreasing the plasma cholesterol levels. On the other hand, it has been investigated that the resveratrol and quercerin (wine polyphenols) [33] and tea cathein [34] are attributed to the up-regulation of LDL receptor activity and subsequently reducing plasma cholesterol level. B-glucan, the active ingredient in oats [35] and calcium supplementation [25] can reduce the reabsorption of bile acids, increasing their excretion into the feces, and thus decreasing the liver and plasma cholesterol. Apple polyphenols [31], garlic [36], and supplementation of red pepper [37] can decrease CETP activity which results in the enhancing the HDL cholesterol concentration. In addition, up regulation of cytochrome $\mathrm{P} 450$ (CYP7A1) activators leads to the reduction of hepatic cholesterol. CYP7A1 is the rate limiting enzyme in the conversion of cholesterol to bile acids in the liver [20]. Studies illustrated that buckwheat [6], curcumin (the main ingredient of turmeric) [38], and grape seed polyphenols [39] can reduce plasma cholesterol level by upregulating the gene of CYP7A1 and increasing bile acid excretion. Despite the positive effects of the above agents, the inhibition of HMG-CoA reductase is known to be the most effective approach to reduce serum cholesterol level $[13,40]$.

\section{HMG-CoA Reductase}

Hydroxymethylglutaryl-coenzymeA reductase (HMG-CoA reductase) catalyzes the conversion of hydroxymethylglutaryl-coenzymeA (HMG-CoA) into mevalonate, the rate-determining step in cholesterol biosynthesis $[16,17]$. Generally, this enzyme has a short half-life, about 3 hours, and higher cholesterol level decreases even its half-life [18]. Two major classes of HMG-CoA reductase are known: the class I enzyme which is present in eukaryotes and some prokaryotes, and located in the endoplasmic reticulum, such as HMGRh in humans; and the class II enzyme which is found in the majority of the archaebacteria and certain eubacteria such as HMGRp in soil bacterium Pseudomonas mevalonii. This group of enzyme is located in cytoplasm [41,42]. The human HMG-CoA reductase gene is located on chromosome 5 and its length is over $24.8 \mathrm{~kb}$ [43]. On the other hand, plants contain different HMG-CoA reductase isoforms that seems to be important in directing the flux of pathway intermediates into specific isoprenoid compounds [44]. Plant HMG-CoA reductase has been localized mainly at the endoplasmic reticulum membrane, but HMG-CoA 
reductase activity has been reported at other sub-cellular regions including plastids, and mitochondria [7,45]. HMGCoA reductase is composed of four regions: the N-terminal region, the conserved membrane domain, the linker region, and the catalytic (or cytosolic) domain. Comparing the primary sequences of genes encoding plant HMG-CoA reductases to those of other eukaryotes shows that the membrane and catalytic domain are highly conserved, whereas the $\mathrm{N}$-terminal and the linker regions are highly divergent $[7,45]$. The main difference in protein structure of HMG-CoA reductases in plants and animals is in the membrane domains which consist of two spans in the membrane domain in plants compared to eight spanning sites in animals (Figure 3) [12,44].

Multigene families are encoding HMG-CoA reductase in plants, two genes in arabidopsis (Arabidopsis thaliana) [45], four genes in tomato [46], and at least 3 genes in potato [47], whereas this enzyme is encoded by a single gene in mammals [48]. Two different HMG-CoA reductase genes (HMG1 and HMG2) in arabidopsis (Arabidopsis thaliana) encode three HMG-CoA reductases isoforms (HMGR1S, HMGR1L, and HMGR2) [44]. It has been demonstrated that the HMG1 is highly expressed in all tissues, whereas the expression of HMG2 gene is restricted to young seedlings, roots and inflorences [45].

\subsection{HMG-CoA Reductase Regulation}

HMG-CoA Reductase is regulated by four different mechanisms, including feedback inhibition, control of gene expression, rate of enzyme degradation and phosphorylation-dephosphorylation $[49,50]$. HMG is a water soluble product which other methabolic pathways can break down when HMG-CoA reductase is inhibited. For this reason, there is no accumulation of potentially toxic precursors, and this phenomenon makes the HMG-CoA reductase an attractive candidate of cholesterol-lowering drugs for reducing low density lipoproteins (LDL-C) and eventually reducing CVD [17].

In plants, developmental signals can control the activity of the HMG-CoA reductase enzyme. HMG-CoA reductase has higher activity in the early stages of plant development and in the rapidly growing parts of plants, such as apical buds and roots. In contrast, the least activity of this enzyme is in mature tissues [51-53]. Also, HMG-CoA reductase activity in plants is regulated by different environmental signals. For example, light can control the HMG-CoA reductase activity at the level of mRNA [54-56]. Also, pathogen-derived elicitor compounds can increases the level of the HMG-CoA reductase activity of those plants that synthesize isoprenoidphytoalexins as part of their defense. For instance, although, the level of HMG-CoA reductase activity in sweet potato root is very low, its activity is increased
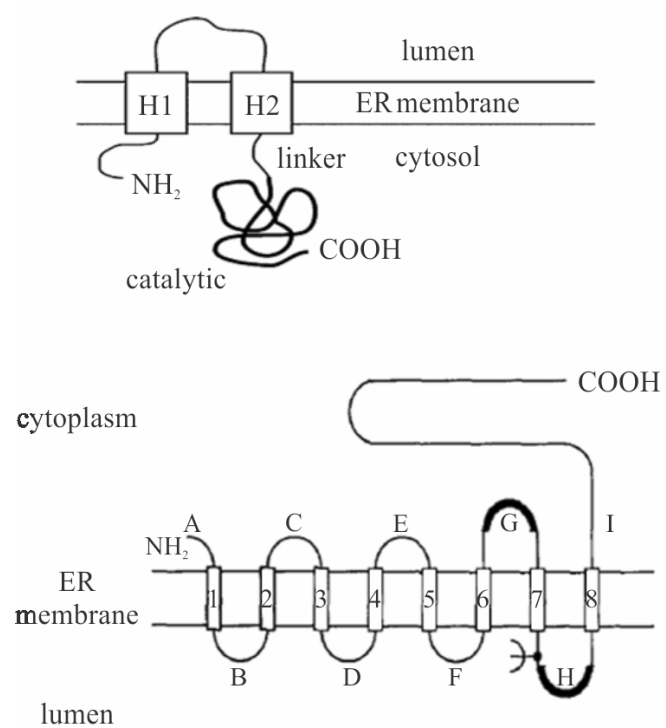

Figure 3. Topological model proposed for (A) plant HMGR and (B) animal HMGR in the ER membrane [12,44].

rapidly after infection by a fungal pathogen $[57,58]$.

The regulatory role of sterols (cholesterol and/or oxysterols) has been demonstrated as they act as inhibitors of HMG-CoA reductase $[8,59,60]$. There are sterol regulatory elements (SRE-1) in the 5' flanking regions of the genes encoding for HMG-CoA synthase, HMG-CoA reductase and the LDL receptor. In the LDL receptor gene as well as HMG-CoA synthase, the SRE-1 is a conditional positive element that increases transcription in the absence of sterols but they do not affect the basal level of transcription when they are present. In contrast, it is suggested that the single SRE-1 in the promoter of HMGCoA reductase repress when sterols are present. Recognition of SRE-1 in the proximal promoter of the aforementioned genes eventually leads to the purification and cloning of a family of transcription factors, termed SRE-binding proteins (SREBPs), which bind to the SRE1 [5]. Two different SREBP genes (SREBP-1 and SREBP-2) coding for three proteins (SREBP-1a, SREBP-1c, and SREBP-2). SREBP-1a and SREBP-1c are formed from one gene using two promoters and different first exons. The aforementioned proteins are different in length and strength of their activation domains. When the cellular sterol levels decrease, mature SREBP $(68 \mathrm{kDa})$ are released from endoplasmic reticulum by using three additional proteins, the Site- 1 and Site- 2 proteases and SCAP (SREBP cleavage-activating protein) [5]. In addition, it has been demonstrated that insulin can up-regulate the HMG-CoA reductase while glucagon down regulates it [18]. Moreover, protein kinase catalyzes the inactivation of HMG-CoA reductase by phosphorylation. Because 
HMG-COA reductase is most active in its unmodified form, phosphorylation decreases its activity [7].

\subsection{Inhibition of HMG-Coa Reductase}

It has been demonstrated that the inhibition of HMG$\mathrm{CoA}$ reductase leads to the activation of transcription factor (SREBP) that promotes expression of the LDL receptor gene, subsequently raising the production of LDL receptors, and thus decreasing the LDL-C level in systematic circulation [61].

Red yeast rice is a typical food that can effectively reduce the blood cholesterol level by inhibiting the HMGCoA reductasein people with hyperlipidemia [62]. It has been demonstrated that cholestine could directly inhibits HMG-CoA reductase followed by the reduction of cholesterol synthesis in hepatic cells (HepG2) [61]. Studies demonstrated that some biologically active compounds derived from mevalonate pathway inhibits the synthesis and enhances the degradation of HMG-CoA Reductase [5]. Fungi contains potent reductase inhibitors, including compactin and mevinolin that block mevalonate synthesis by inhibiting the HMG-CoA reductase as well as 200 -fold increase in the reductase protein abundance (inactive form because it is blocked by the inhibitor) in few hours. The 200-fold increase could be the result of the eightfold increase in initiation of transcription (effect of sterol agent), fivefold increase in mRNA translation (effect of non-sterol agent) and fivefold decrease in enzyme molecule degradation (effect of both sterol and non-sterol agent) [8].

Seventeen brands of seven major cholesterol-lowering drug classes are known in the world, which are the HMG-CoA reductase inhibitors (Statins, such as compactin, simvestatin, atorvestatin, etc). These are the most prescribed group of drugs spread throughout the world in treating hypercholesterolemia [63]; however application of this group of drugs has side effects including rashes and gastrointestinal symptoms [20]. Statins exist in two types: Type 1 statins which are fungal products such as lovastatin (Mevacor ${ }^{\mathbb{Q}}$, mevinolin), and simvastatin (Zo$\left.\operatorname{cor}^{\mathbb{B}}\right)$; and type II which are completely synthetic such as atrovastatin $\left(\right.$ Lipitor $\left.^{\circledR}\right)$, fluvastatin $\left(\right.$ Lescol $\left.^{\circledR}\right)$ [40].

Isopronoid derived from plant isoprenoid pathways could suppress HMG-CoA reductase activity by modulating mRNA translation and the proteolitic degradation. In addition, it has been proposed that aforementioned post-transcriptional procedures are activated directly by acyclic terpenoids and indirectly by cyclic terpenoids [50]. HMG-CoA reductase in vivo in the rat can be inhibited up to $70 \%$ by the cyclic monoterpenes as cineole and menthol [64]. Corell et al. (1994) illustrated that farnesol as non-sterol derivatives of mevalonate pathway accelerates the degradation of reductase in animal cells
[1], and Elson et al. (1999) suggested it as a key player in the post-translational control of reductase [50]. In contrast, farnesol treatment in tobacco bright yellow-2 Cells (TBY-2) increases the microsomal HMG-CoA reductase activity; however, its effect is concentration dependent [65]. Also, it has been reported that Plantago asiatica essential oils can suppress the expression of HMG-CoA reductase in vitro and in vivo [66]. In addition, tocotrienols, a group of Vitamin E stereoisomers containing farnesylisoprenoid tail, are a group of HMG-CoA reductase inhibitors that are abundant in rice bran and barely [67].

\section{Concluding Remarks: Current State and Potential Future Development}

The knowledge emerging from the earlier studies reviewed above enables the characterization of some plant biologically active compounds which can inhibit the synthesis and enhance the degradation of HMG-CoA reductase. Cardiovascular disease (CVD) is the main cause of death in industrial countries. Statins are known to be the main treatments for CVD [63]; however the application of this group of drugs, next to their high cost [68], has shown side effects including rashes and gastrointestinal symptoms [69]. For these reasons, there is a necessity to examine the viability of other biologically active natural products as an alternative to statin treatment. In the same relation, an investigation to the essential oils (EOs) may be worthwhile as they are natural products which generally are obtained from flowers, leaves or other parts of plant, and composed of complex mixture of isoprenoids, polyketides and very few alkaloids. They have versatile and varying applications worldwide including folk medicine, Chinese medicine, alternative medicine, aromatherapy, as well as in the cosmetics and perfumes industries, and in food flavoring and cleaning products [70-73]. It is believed that the investigation of different plants essential oils can eventually lead to a discovery of new means for the treatment of hypercholesterolemia and cardiovascular disease (CVD) using natural products.

\section{Acknowledgements}

Thanks are due to Dr. Soheil. S. Mahmoud, the University of British Columbia Okanagan, for his feedback and useful discussions. Financial support from Fonds de Recherche du Québec Nature et Technologies (FQRNT) is greatly acknowledged.

\section{REFERENCES}

[1] C. C. Correll, L. Ng and P. A. Edwards, "Identification of Farnesolas the Nonsterol Derivative of Mevalonic Acid Required for the Accelerated Degradation of 3-Hydroxy-3- 
Methylglutaryl-Coenzyme A Reductase," Journal of Biological Chemistry, Vol. 269, No. 7, 1994, pp. 1739017393.

[2] S. A. Holstein and R. J. Hohl, "Isoprenoids: Remarkable Diversity of Form and Function," Lipids, Vol. 39, No. 4, 2004, pp. 293-309. doi:10.1007/s11745-004-1233-3

[3] Y. G. Lin, M. A. Vermeer and E. A. Trautwein, "Triterpenic Acids Present in Hawthorn Lower Plasma Cholesterol by Inhibiting Intestinal ACAT Activity in Hamsters," Evidence-Based Complementary and Alternative Medicine, Vol. 2011, 2011, Article ID 801272. doi:10.1093/ecam/nep007

[4] P. M. Dewick, "Medicinal Natural Products," John Wiley \& Sons, Chichester, 2009. doi:10.1002/9780470742761

[5] P. A. Edwards, "Sterols and Isoprenoids: Signaling Molecules Derived from the Cholesterol Biosynthetic Pathway," Annual Review of Biochemistry, Vol. 69, 1999, pp. 157-185. doi:10.1146/annurev.biochem.68.1.157

[6] T. Kuwabara, K. H. Han, N. Hashimoto, H. Yamauchi, K. Shimada, M. Sekikawa and M. Fukushima, "Tartary Buckwheat Sprout Powder Lowers Plasma Cholesterol Level in Rats," Journal of Nutritional Science and Vitaminology. Vol. 53, No. 6, 2007, pp. 501-507. doi:10.3177/jnsv. 53.501

[7] B. A. Stermer, G. M. Bianchini and K. L. Korth, "Regulation of HMG-COA Reductase Activity in Plants," Journal of Lipid Research, Vol. 35, No. 7, 1994, pp. 11331140.

[8] S. C. Hashimoto, A. Drevon, D. B. Weinstein, J. S. Bernett, S. Dayton and D. Steinberg., "Activity of Acyl-CoACholesterol Acyltransferaseand 3-Hydroxy-3-Methylglutaryl-CoA Reductase in Subfractions of Hepatic Microsomes Enriched with Cholesterol," Biochimica Et BiophysicaActa, Vol. 754, No. 2, 1983, pp. 126-133. doi:10.1016/0005-2760(83)90153-4

[9] E. Cordoba, M. Salmi and P. Leon, "Unravellingthe Regulatory Mechanisms that Modulate the MEP Pathway in Higher Plants," Journal of Experimental Botany, Vol. 60, No. 10, 2009, pp. 2933-2943. doi:10.1093/jxb/erp190

[10] Y. V. Ershov, "2-C-Methylerythritol Phosphate Pathway of Isoprenoid Biosynthesis as a Target in Identifying New Antibiotics, Herbicides, and Immunomodulators: A Review," Applied Biochemistry and Microbiology, Vol. 43, 2007, pp. 115-138. doi:10.1134/S0003683807020019

[11] M. Rohmer, "The Discovery of a Mevalonate-Independent Pathway for Isoprenoid Biosynthesis in Bacteria, Algae and Higher Plants," Natural Product Reports, Vol. 16, No. 5, 1999, pp. 565-574. doi:10.1039/a709175c

[12] J. Roitelman, E. H. Olender, S. Bar-Nun, J. William A. Dunn and R. D. Simoni, "Immunological Evidence for Eight Spans in the Membrane Domain of 3-Hydroxy-3Methylglutaryl Conzyme A Reductase: Implications for Enzyme Degradation in the Endoplasmic Reticulum," Rockefeller University Press, Rockefeller, 1992, pp. 959973. doi:10.1083/jcb.117.5.959

[13] Y. Chen, "Cholesterol-Lowering Nutraceuticalsand Functional Foods," Journal of Agricultural and Food Chemistry, Vol. 56, No. 19, 2008, pp. 8761-8773.

\section{doi:10.1021/jf801566r}

[14] J. Kirby and J. D. Keasling, "Biosynthesis of Plant Isoprenoids: Perspectives for Microbial Engineering," Annual Review of Plant Biology, Vol. 60, 2009, pp. 335-355. doi:10.1146/annurev.arplant.043008.091955

[15] Bjorkelid, T. Bergfors, L. M. Henriksson, A. L. Stern, T. Unge, S. L. Mowbray and T. A. Jones, "Structural and Functional Studies of Mycobacterial IspD Enzymes," Acta Crystallographica Section D: Biological Crystallography, Vol. 67, 2011, pp. 403-414. doi:10.1107/S0907444911006160

[16] E. J. Park, D. Lee, Y. G. Shin, D. D. Lantvit, R. B. van Breemen, A. D. Kinghorn and J. M. Pezzuto, "Analysis of 3-Hydroxy-3-Methylglutaryl-Coenzyme A Reductase Inhibitors Using Liquid Chromatography-Electrospray Mass Spectrometry," Journal of Chromatography, Vol. 754, No. 2, 2001, pp. 327-332. doi:10.1016/S0378-4347(00)00620-4

[17] K. Vonbergmann, A. Beck, C. Engel and O. Leiss, “Administration of a Terpene Mixture Inhibits Cholesterol Nucleation in Bile from Patients with Cholesterol Gallstones," Klinische Wochenschrift, Vol. 65, No. 10, 1987, pp. 458-462. doi:10.1007/BF01712838

[18] D. Rozman and M. R. Waterman, "Sterol Biosynthesis" In: J. I. Mason, Ed., Genetics of Steroid Biosynthesis and Function, 2002. http:// www.eBookstore.tandf.co.uk

[19] E. Oldfield, "Targeting Isoprenoid Biosynthesis for Drug Discovery: Bench to Bedside," Accounts of Chemical Research, Vol. 43, No. 9, 2010, pp. 1216-1226. doi:10.1021/ar100026v

[20] Z. Y. Chen, K. Y. Ma, Y. T. Liang, C. Peng and Y. Y. Zuo, "Role and Classification of Cholesterol-Lowering Functional Foods," Journal of Functional Foods, Vol. 3, No. 2, 2011, pp. 61-69. doi:10.1016/i.jff.2011.02.003

[21] A. Atzel and J. R. Wetterau, "Mechanism of Microsomal Triglyceride Transfer Protein Catalyzed Lipid Transport," Biochemistry, Vol. 32, No. 39, 1993, pp. 10444-10450. doi:10.1021/bi00090a021

[22] H. R. Davis, L. J. Zhu, L. M. Hoos, G. Tetzloff, M. Maguire, J. J. Liu, X. R. Yao, S. P. N. Iyer, M. H. Lam, E. G. Lund, P. A. Detmers, M. P. Graziano and S. W. Altmann, "Niemann-Pick C1 Like 1 (NPC1L1) Is the Intestinal Phytosterol and Cholesterol Transporter and a Key Modulator of Whole-Body Cholesterol Homeostasis," Journal of Biological Chemistry, Vol. 279, 2004, pp. 33586-33592. doi:10.1074/jbc.M405817200

[23] K. A. Do, A. Green, J. R. Guthrie, E. C. Dudley, H. G. Burger and L. Dennerstein, "Longitudinal Study of Risk Factors for Coronary Heart Disease Across the Menopausal Transition," American Journal of Epidemiology, Vol. 151, No. 6, 2000, pp. 584-593. doi:10.1093/oxfordjournals.aje.a010246

[24] K. Y. Ma, N. Yang, R. Jiao, C. Peng, L. Guan, Y. Huang, and Z. Y. Chen, "Dietary Calcium Decreases Plasma Cholesterol by Down-Regulation of Intestinal NiemannPick C1 Like 1 and Microsomal Triacylglycerol Transport Protein and Up-Regulation of CYP7A1 and ABCG 5/8 in Hamsters," Molecular Nutrition \& Food Research, 
Vol. 55, No. 2, 2011, pp. 247-258.

doi:10.1002/mnfr.201000161

[25] C. Messa, M. Notarnicola, F. Russo, A. Cavallini, V. Pallottini, A. Trentalance, M. Bifulco, C. Laezza and M. G. Caruso, "Estrogenic Regulation of Cholesterol Biosynthesis and Cell Growth in DLD-1 Human Colon Cancer Cells," Scandinavian Journal of Gastroenterology, Vol. 40, No. 12, 2005, pp. 1454-1461. doi:10.1080/00365520510024007

[26] P. Mirmiran, N. Noori, M. B. Zavareh and F. Azizi, "Fruit and Vegetable Consumption and Risk Factors for Cardiovascular Disease," Metabolism-Clinical and Experimental, Vol. 58, No. 4, 2009, pp. 460-468. doi:10.1016/j.metabol.2008.11.002

[27] H. Suido, T. Tanaka, T. Tabei, A. Takeuchi, M. Okita, T. Kishimoto, S. Kasayama and K. Higashino, "A Mixed Green Vegetable and Fruit Beverage Decreased the Serum Level of Low-Density Lipoprotein Cholesterol in Hypercholesterolemic Patients," Journal of Agricultural and Food Chemistry, Vol. 50, No. 11, 2002, pp. 33463350. doi:10.1021/jf0116698

[28] S. W. Andersson, J. Skinner, L. Ellegard, A. A. Welch, S. Bingham, A. Mulligan, H. Andersson and K. T. Khaw, "Intake of Dietary Plant Sterols Is Inversely Related to Serum Cholesterol Concentration in Men and Women in the EPIC Norfolk Population: A Cross-Sectional Study," European Journal of Clinical Nutrition, Vol. 58, 2004, pp. 1378-1385. doi:10.1038/sj.ejen. 1601980

[29] M. B. Katan, S. M. Grundy, P. Jones, M. Law, T. Miettinen, R. Paoletti and P. Stresa Workshop, "Efficacy and Safety of Plant Stanols and Sterols in the Management of Blood Cholesterol Levels," Mayo Clinic Proceedings, Vol. 78, No. 8, 2003, pp. 965-978. doi:10.4065/78.8.965

[30] R. P. Mensink and M. B. Katan, "Effect of Dietary Fatty Acids on Serum Lipids and Lipoproteins. A MetaAnalysis of 27 Trials," Arteriosclerosis, Thrombosis, and Vascular Biology, Vol. 12, No. 8, 1992, pp. 911-919.

[31] C. K. Lam, J. N. Chen, Y. Cao, L. Yang, Y. M. Wong, S. Y. V. Yeung, X. Q. Yao, Y. Huang, and Z. Y. Chen, "Conjugated and Non-Conjugated Octadecaenoic Acids Affect Differently Intestinal Acyl Coenzyme A: Cholesterol Acyltransferase Activity," Atherosclerosis, Vol. 198, No. 1, 2008, pp. 85-93.

doi:10.1016/j.atherosclerosis.2007.11.001

[32] M. S. Lee, J. Y. Park, H. Freake, I. S. Kwun and Y. Kim, "Green Tea Catechin Enhances Cholesterol 7 Alpha-Hydroxylase Gene Expression in HepG2 Cells," The British Journal of Nutrition, Vol. 99, No. 6, 2008, pp. 1182-1185. doi:10.1017/S0007114507864816

[33] S. Pal, N. Ho, C. Santos, P. Dubois, J. Mamo, K. Croft, and E. Allister, "Red Wine Polyphenolics Increase LDL Receptor Expression and Activity and Suppress the Secretion of ApoB100 from Human HepG2 Cells," Journal of Nutrition, Vol. 133, No. 3, 2003, pp. 700-706.

[34] C. A. Bursill, M. Abbey and P. D. Roach, "A Green Tea Extract Lowers Plasma Cholesterol by Inhibiting Cholesterol Synthesis and Up Regulating the LDL Receptor in the Cholesterol-Fed Rabbit," Atherosclerosis, Vol. 193, No. 1, 2007, pp. 86-93. doi:10.1016/j.atherosclerosis.2006.08.033

[35] H. J. Kim and P. J. White, "In Vitro Bile-Acid Binding and Fermentation of High, Medium, and Low Molecular Weight Beta-Glucan," Journal of Agricultural and Food Chemistry, Vol. 58, No. 1, 2010, pp. 628-634. doi:10.1021/jf902508t

[36] M. J. Kwon, Y. S. Song, M. S. Choi, S. J. Park, K. S. Jeong and Y. O. Song, "Cholesteryl Ester Transfer Protein Activity and Atherogenic Parameters in Rabbits Supplemented with Cholesterol and Garlic Powder," Life Sciences, Vol. 72, No. 26, 2003, pp. 2953-2964. doi:10.1016/S0024-3205(03)00234-0

[37] M. J. Kwon, Y. S. Song, M. S. Choi and Y. O. Song, "Red Pepper Attenuates Cholesteryl Ester Transfer Protein Activity and Atherosclerosis in Cholesterol-Fed Rabbits," Clinica Chimica Acta, Vol. 332, No. 1-2, 2003, pp. 37-44. doi:10.1016/S0009-8981(03)00118-9

[38] M. Kim and Y. Kim, "Hypocholesterolemic Effects of Curcumin via Up-Regulation of Cholesterol 7a-Hydroxylase in Rats Fed a High Fat Diet," Nutrition Research and Practice, Vol. 4, No. 3, 2010, pp. 191-195. doi:10.4162/nrp.2010.4.3.191

[39] R. Jiao, Z. S. Zhang, H. J. Yu, Y. Huang and Z. Y. Chen, "Hypocholesterolemic Activity of Grape Seed Proanthocyanidin is Mediated by Enhancement of Bile Acid Excretion and Up-Regulation of CYP7A1," Journal of $\mathrm{Nu}$ tritional Biochemistry, Vol. 21, No. 11, 2010, pp. 11341139. doi:10.1016/i.jnutbio.2009.10.007

[40] N. Singh, J. Tamariz, G. Chamorro and J. L.MedinaFranco, "Inhibitors of HMG-CoA Reductase: Current and Future Prospects," Medicinal Chemistry, Vol. 9, No. 11, 2009, pp. 1272-1283. doi:10.2174/138955709789878105

[41] D. A. Bochar, C. V. Stauffacher and V. W. Rodwell, "Sequence Comparisons Reveal Two Classes of 3-Hydroxy-3-Methylglutaryl Coenzyme A Reductase," Molecular Genetics and Metabolism, Vol. 66, No. 2, 1999, pp. 122-127. doi:10.1006/mgme.1998.2786

[42] L. Tabernero, V. W. Rodwell and C. V. Stauffacher, "Crystal Structure of a Statin Bound to a Class II Hydroxy-Methylglutaryl-CoA Reductase," Journal of Biological Chemistry, Vol. 278, No. 22, 2003, pp. 19933 19938. doi:10.1074/jbc.M213006200

[43] J. A. Friesen and V. W. Rodwell, "The 3-Hydroxy-3Methylglutaryl Coenzyme-A (HMG-CoA) Reductases," Genome Biology, Vol. 5, No. 11, 2004, p. 248. doi: $10.1186 / \mathrm{gb}-2004-5-11-248$

[44] P. Leivar, V. M. Gonzalez, S. Castel, R. N. Trelease, C. Lopez-Iglesias, M. Arro, A. Boronat, N. Campos, A. Ferrer and X. Fernandez-Busquets, "Subcellular Localization of Arabidopsis 3-Hydroxy-3-Methylglutaryl-Coenzyme A Reductase," Plant Physiology, Vol. 137, No. 1, 2005, pp. 57-69. doi:10.1104/pp.104.050245

[45] N. Cunillera, M. Arro, D. Delourme, F. Karst, A. Boronat and A. Ferrer, "Arabidopsis thaliana Contains Two Differentially Expressed Farnesyl-Diphosphate Synthase Genes," Journal of Biological Chemistry, Vol. 271, No. 13, 1996, pp. 7774-7780. doi:10.1074/jbc.271.13.7774

[46] J. G. Jelesko, S. M. Jenkins, M. Rodriguez-Concepcion 
and W. Gruissem, "Regulation of Tomato HMG1 during Cell Proliferation and Growth," Planta, Vol. 208, No. 3, 1999, pp. 310-318. doi:10.1007/s004250050564

[47] Y. M. Crane and K. L. Korth, "Regulated Accumulation of 3-Hydroxy-3-Methylglutaryl CoA Reductase Protein in Potato Cell Cultures: Effects of Calcium and Enzyme Inhibitors," Journal of Plant Physiology, Vol. 159, No. 12, 2002, pp. 1301-1307. doi:10.1078/0176-1617-00874

[48] V. Lumbreras, N. Campos and A. Boronat, "The Use of an Alternative Promoter in the Arabidopsis thaliana HMG1 Gene Generates a Messenger-RNA that Encodes a Novel 3-Hydroxy-3-Methylglutaryl Coenzyme-A Reductase Isoform with an Extended N-Terminal Region," Plant Journal, Vol. 8, No. 4, 1995, pp. 541-549. doi:10.1046/j.1365-313X.1995.8040541.X

[49] P. A. Edwards and J. Ericsson, "Signaling Molecules Derived from the Cholesterol Biosynthetic Pathway: Mechanisms of Action and Possible Roles in Human Disease," Current Opinion in Lipidology, Vol. 9, No. 5, 1998, pp. 433-440. doi:10.1097/00041433-199810000-00007

[50] C. E. Elson, D. M. Peffley, P. Hentosh and H. B. Mo, "Isoprenoid-Mediated Inhibition of Mevalonate Synthesis: Potential Application to Cancer," Proceedings of the Society for Experimental Biology and Medicine, Vol. 221, No. 4, 1999, pp. 294-311. doi:10.1046/j.1525-1373.1999.d01-87.x

[51] T. J. Bach, D. H. Rogers and H. Rudney, "DetergentSolubilization, Purification, and Characterization of Membrane-Bound 3-Hydroxy-3-Methylglutaryl-CoenzymeA Reductase from Radish Seedlings," European Journal of Biochemistry, Vol. 154, No. 1, 1986, pp. 103-111. doi:10.1111/j.1432-1033.1986.tb09364.x

[52] J. D. Brooker and D. W. Russell, "Properties of microsomal 3-Hydroxy-3-Methylglutaryl Coenzyme A Reductase from Pisum-Sativum Seedlings," Archives of Biochemistry and Biophysics, Vol. 167, No. 2, 1975, pp. 723729. doi:10.1016/0003-9861(75)90517-2

[53] J. O. Narita and W. Gruissem, "Tomato Hydroxymethylglutaryl-CoA Reductase Is Required Early in Fruit Development but not During Ripening," Plant Cell, Vol. 1, No. 2, 1989, pp. 181-190. doi:10.2307/3869034

[54] M. Gleizes, G. Pauly, C. Bernarddagan and R. Jacques, "Effects of Light on Terpene Hydrocarbon Synthesis in Pinus-Pinaster," Physiologia Plantarum, Vol. 50, No. 1, 1980, pp. 16-20. doi:10.1111/j.1399-3054.1980.tb02676.x

[55] B. R. Loveys and P. F. Wareing, "Red Light Controlled Production of Gibberllin in Etiolated Wheat Leaves," Planta, Vol. 98, No. 2, 1971, pp. 109-116. doi:10.1007/BF00385343

[56] S. Tanaka, T. Yamaura, R. Shigemoto and M. Tabata, "Phytochrome-Mediated Production of Monoterpenes in Thyme Seedlings," Phytochemistry, Vol. 28, No. 11, 1989, pp. 2955-2957. doi:10.1016/0031-9422(89)80260-2

[57] B. A. Stermer and R. M. Bostock, "Involvement of 3Hydroxy-3-Methylglutaryl Coenzyme-A Reductase in the Regulation of Sesquiterpenoid Phytoalexin Synthesis in Potato," Plant Physiology, Vol. 84, No. 2, 1987, pp. 404-

\section{8. doi:10.1104/pp.84.2.404}

[58] H. Suzuki, K. Oba and I. Uritani, "Occurrence and Some Properties of 3-Hydroxy-3-Methylglutaryl Coenzyme A Reductase in Sweet-Potato Roots Infected by Ceratocystis Fimbriata," Physiological Plant Pathology, Vol. 7, No. 3, 1975, pp. 265-276. doi:10.1016/0048-4059(75)90031-4

[59] B. I. Cohen, R. F. Raicht and E. H. Mosbach, "Sterol Metabolism Studies in the Rat. Effects of Dietary Plant Sterols and Bile Acids on Sterol Metabolism," Biochimica Et Biophysica Acta, Vol. 487, No. 2, 1977, pp. $287-$ 296. doi:10.1016/0005-2760(77)90005-4

[60] L. L. Smith and B. H. Johnson, "Biological-Activities of Oxysterols," Free Radical Biology and Medicine, Vol. 7, No. 3, 1989, pp. 285-332.

doi:10.1016/0891-5849(89)90136-6

[61] J. D. Horton, J. L. Goldstein and M. S. Brown, "SREBPs: Activators of the Complete Program of Cholesterol and Fatty Acid Synthesis in the Liver," Journal of Clinical Investigation, Vol. 109, No. 9, 2002, pp. 1125-1131. doi:10.1172/JCI200215593

[62] J. X. Wang, Z. L. Lu, J. M. Chi, W. H. Wang, M. Z. Su, W. R. Kou, P. L. Yu, L. J. Yu, J. S. Zhu and J. Chang, "Multicenter Clinical Trial of the Serum Lipid-Lowering Effects of a Monascus Purpureus (Red Yeast) Rice Preparation from Traditional Chinese Medicine," Current Therapeutic Research-Clinical and Experimental, Vol. 58, No. 12, 1997, pp. 964-978. doi:10.1016/S0011-393X(97)80063-X

[63] A. Leszczynska, B. Burzynska, D. Plochocka, J. Kaminska, M. Zimnicka, M. Kania, M. Kiliszek, M. WysockaKapcinska, W. Danikiewicz and A. Szkopinska, "Investigating the Effects of Statins on Cellular Lipid Metabolism Using a Yeast Expression System," PloS ONE, Vol. 4, No. 12, 2009, Article ID: e8499. doi:10.1371/journal.pone.0008499

[64] R. J. Clegg, B. Middleton, G. D. Bell and D. A. White, "The Mechanism of Cyclic Monoterpene Inhibition of Hepatic 3-Hydroxy-3-Methylglutaryl Coenzyme-A Reductase in Vivo in the Rat," Journal of Biological Chemistry, Vol. 257, No. 5, 1982, pp. 2294-2299.

[65] A. Hemmerlin and T. J. Bach, "Farnesol-Induced Cell Death and Stimulation of 3-Hydroxy-3-Methylglutaryl -Coenzyme A Reductase Activity in Tobacco cv Bright Yellow-2 Cells," Plant Physiology, Vol. 123, No. 4, 2000, pp. 1257-1268. doi:10.1104/pp.123.4.1257

[66] M. J. Chung, K. W. Park, K. H. Kim, C. T. Kim, J. P. Baek, K. H. Bang, Y. M. Choi and S. J. Lee, "Asian Plantain (Plantago asiatica) Essential Oils Suppress 3Hydroxy-3-Methylglutaryl-Co-Enzyme A Reductase Expression in Vitro and in Vivo and Show Hypocholesterolaemic Properties in Mice," British Journal of Nutrition, Vol. 99, No. 1, 2008, pp. 67-75. doi: $10.1017 /$ S0007114507798926

[67] S. Das, K. Nesaretnam and D. K. Das, "Tocotrienols in Cardioprotection", In: G. Litwack, Ed., Vitamin E: Vitamins and Hormones Advances in Research and Applications, American Medical Association, Chicago, 2007, p. 419-433. doi:10.1001/jama.1945.02860260070044 
[68] J. L. Goldstein and M. S. Brown, "Regulation of the Mevalonate Pathway," Nature, Vol. 343, No. 6257, 1990, pp. 425-430. doi:10.1038/343425a 0

[69] Z. Y. Chen, K. Y. Ma, Y. Liang, C. Peng and Y. Zuo, "Role and Classification of Cholesterol-Lowering Functional Foods," Journal of Functional Foods, Vol. 3, No. 2, 2011, pp. 61-69. doi:10.1016/j.jff.2011.02.003

[70] C. Dobetsberger and G. Buchbauer, "Actions of Essential Oils on the Central Nervous System: An updated review," Flavour and Fragrance Journal, Vol. 26, No. 5, 2011, pp. 300-316.
[71] D. P. D. Sousa, "Analgesic like Activity of Essential Oils Constituents," Molecules, Vol. 16, No. 3, 2011, pp. 2233 2252. doi:10.3390/molecules 16032233

[72] D. J. Newman, G. M. Cragg and K. M. Snader, "The Influence of Natural Products upon Drug Discovery," Natural Product Reports, Vol. 17, No. 3, 2000, pp. 215234. doi:10.1039/a902202c

[73] Interactive European Network for Industrial Crops and their Applications, "Summary Report for the European Union 2000-2005," IENICA, 2005. 